\title{
Analysis of a Network Bandwidth Status based Distributed Multimedia Streaming Delivery Algorithm across the Heterogeneous Wireless Networks
}

\author{
Ronnie D. Caytiles and Byungjoo Park ${ }^{*}$ \\ Department of Multimedia Engineering, Hannam University \\ 133 Ojeong-dong, Daeduk-gu, Daejeon, Korea \\ rdcaytiles@gmail.com, *bjpark@hnu.kr
}

\begin{abstract}
The mobility support for the integration of heterogeneous wireless networks has been managed by centralized architectures of flow mobility which are susceptible for single point of failures, scalability, and energy efficiency issues. This paper deals with the analysis of the proxy mobile Internet protocol version 6 (PMIPv6) based distributed mobility management (DMM) based integration of heterogeneous wireless networks in order to support a seamless flow distribution of multimedia contents. It aims to provide the ubiquitous environment with a seamless mobility support for disparate distribution of multimedia services. DMM is an emerging paradigm for addressing the issues for centralized mobility support which have distributed mobility anchors taking into consideration the continuously increasing multimedia traffic across the heterogeneous wireless networks. Thus, the DMM based solutions warrants a seamless for the distribution of multimedia services.
\end{abstract}

Keywords: distributed mobility management, wireless networks, flow mobility

\section{Introduction}

A seamless mobility support for connectivity handover between wireless networks is essentially required nowadays for the delivery of real-time multimedia services. The increasing bulk of multimedia traffic has brought the integration and cooperation among heterogeneous access networks in order to cope with such demands. The volume of traffic is projected to be tripled by 2021 based on the complete visual networking index (VNI) forecast [1] last 2016. The continuous growth of the miniaturization of telecommunication technologies into powerful handheld devices led to the complete revolution into a ubiquitous era. In this regard, researchers and scientists have standardized numerous solutions of mobility support in order to address this rapid telecommunication evolution. This is to address the continuously increasing demand for the increased bandwidth of mobile traffic of multimedia services.

The standardized mobility management solutions provided by the Internet Engineering Task Force (IETF) and the $3^{\text {rd }}$ Generation Partnership Project (3GPP) are mostly considering a centralized approach for providing mobility support for the handover of connectivity between different access networks. With these solutions, a central mobility anchor is used to direct traffic which is susceptible to a number of mobility management issues such as low scalability, reliability (i.e., single point of failure), sub-optimal routing, higher packet loss rate, signaling overhead, lack of granularity on the mobility management service, and leads to a more complex

Received (June 8, 2017), Review Result (September 15, 2017), Accepted (September 27, 2017)

* Corresponding Author 
deployment $[2,3]$. Thus, the need for alternative solutions in order to address this issues are essentially important in order to provide the Internet users of a seamless and balanced flow mobility of multimedia services in the ubiquitous environment.

This paper aims to provide a study of the analysis of a proxy mobile Internet protocol version 6 (PMIPv6) based distributed mobility management (DMM) based support for the handover of connectivity among heterogeneous wireless networks. Instead of using a centralized mobility anchor, it utilizes the mobility anchors that are closer to the user mobile devices, wherein the data and control infrastructures are distributed to the entities that are located at the edge of wireless networks. In this regard, traffic is distributed locally to exploit the usage of different access gateways that belong to different wireless network technologies. Thus, the multimedia services can be routed in a very optimal path, leaving the network core offloaded with the distribution and centralized control of traffic.

The rest of this paper is organized as follows: Section 2 provides an overview of why distributed mobility management needs to be adapted in lieu of the centralized mobility solutions; the analysis of the distributed mobility management (DMM) based integration of heterogeneous wireless networks is discussed in Section 3; the analysis of the PMIPv6 based DMM multimedia service delivery is presented in Section 4; and the concluding remarks in Section 5.

\section{Centralized versus Distributed Mobility Management}

Recently, mobile systems are based on hierarchical systems that employs centralized mobility management protocols (e.g., Proxy Mobile Internet Protocol version 6 (PMIPv6) [4, 5], and dual-stack Mobile Internet Protocol version 6 (MIPv6) [6]) in handling mobility support for mobile user devices. The MIPv6 is an IETF standard that allows a mobile user device to maintain its connectivity with its correspondent nodes (CNs) whenever it roams across the heterogeneous wireless networks. The multimedia services provided by its $\mathrm{CN}$ are delivered to the mobile user's device through its home agent (HA) that resides at its home network as shown in the handover process outlined in Figure 1(a). A fixed home address (HoA) is allocated by the HA to the user's mobile device and whenever it moves into a different network, another address in the form of care-of address $(\mathrm{CoA})$ is allocated. The CoA serves as the temporary address user's mobile device when it is away with its home network which makes it reachable for its CNs [6]. However, the mobility support provided by MIPv6 suffers from major setbacks of higher handover latency, higher packet loss, and signaling overheads.

The PMIPv6 is a network-based mobility support standardized by the Networkbased Localized Mobility Management (NETLMM) working group of the Internet Engineering Task Force (IETF) in order to address the signaling overhead concerns of MIPv6 [4]. The mobile user devices need not to participate in the mobility related signaling whenever it roams between wireless networks within the PMIPv6 domain. The mobility management functions are provided by the network entities in lieu of the user mobile devices, thus, the users become independent of its movement. The handover process for PMIPv6 is depicted in Figure 1(b) wherein the network entities were made responsible for the mobility management support for user mobile devices. 


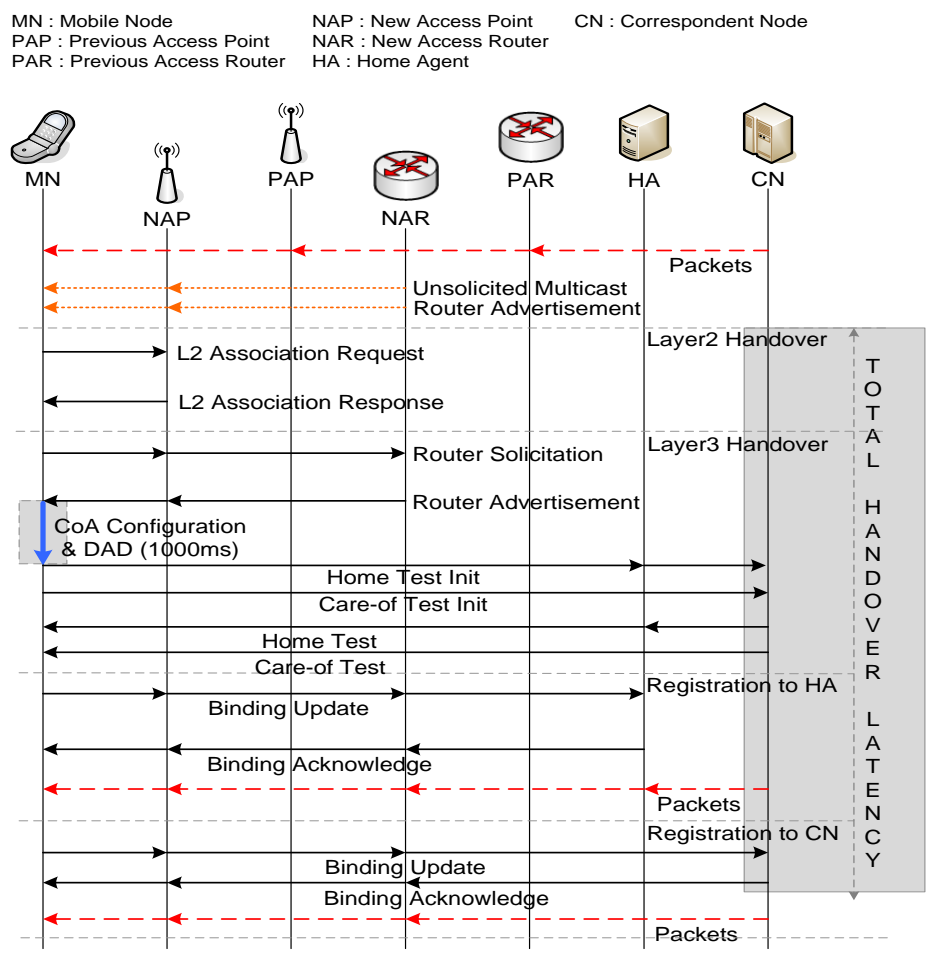

(a) MIPv6 Handover Process

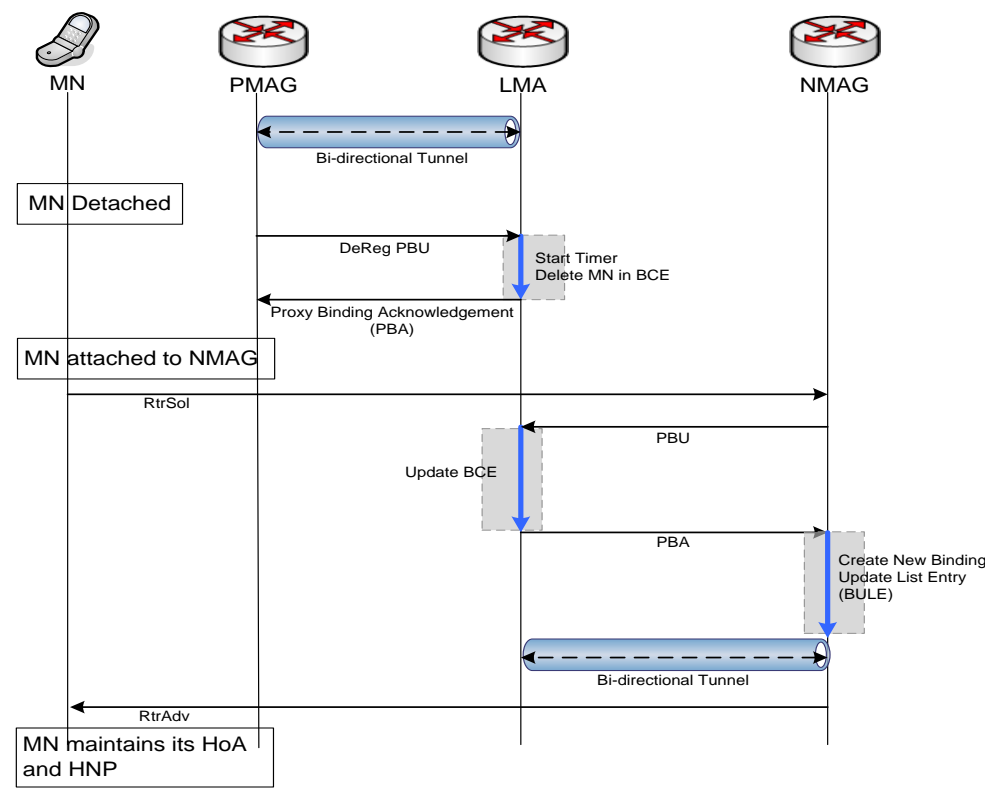

(b) PMIPv6 Handover Process indicating the MN's movement to a new MAG

\section{Figure 1. The Centralized Mobility Management Handover Operations}

The proxy mobility agents of PMIPv6 perform the necessary mobility signaling initializations as the mobile user device moves from one wireless network to the other. These mobility agents are: (1) the mobility access gateway (MAG), which performs the mobility related signaling operations for the mobile user device that are currently attached into its access points; and (2) the local mobility anchor (LMA), which maintains the collection of IP address of all MNs attached within the localized mobility domain (LMD). 
The MAG is residing in one of the access routers (AR) and is responsible for mobile user device' movement detection, routing states coordination, and IP connectivity provision. The LMA is residing in the mobile user device' home network and is acting as the local HA. A bidirectional tunnel is created between the LMA and MAG as soon as the mobile user device attaches to the MAG. The LMA intercepts the multimedia services that are intended to the mobile user device and tunnels them towards the MAG to which its connectivity is attached. And locally, the MAG decapsulates the received packets and forward it to the mobile user device.

The LMA in PMIPv6 and HA in MIPv6 are acting as the centralized mobility anchor for the movement of the mobile user device across the heterogeneous wireless networks as well as for the delivery of multimedia services. They serve as the central controller for the distribution of traffic between interlaced wireless access networks. This mobility management support is considered a sensible approach for managing the traffic flow for the existing integration of wireless networks. However, based on the analysis for centralized mobility management systems, the following limitations have been identified whenever the increasing volume of multimedia traffic is considered [7]:

(1) Low scalability. The centralized mobility anchors are required to have processing and routing capabilities for the mobile user device's traffic.

(2) Reliability. A single point of failure is the main concern for centralized mobility anchors (LMA, HA).

(3) Suboptimal routing. Traffic is always navigated through the centralized mobility anchors which are normally residing at the mobile user device' home link. Thus, the multimedia services traverse a longer path between the mobile user device and its CNs in general. There are times that the traffic needs to go through the home network before it is forwarded to the current location of the mobile user device which leads to unnecessary delays and consumption of network resources.

(4) Higher packet loss rate. The congestion of multimedia traffic on the centralized mobility anchors could trigger a higher rate of packet losses. In real-time multimedia services as well as on online gaming applications, user experience is greatly affected by packet loss.

(5) Signaling overhead. The mobile user device and network entities are required for exchanging a number of mobility management signaling in order to ensure the handover between a wireless network and the other across the integrated heterogeneous wireless networks. Such mechanisms are adding significant time for the handover of connectivity between wireless networks. Thus, the delivery of multimedia services to the users can be greatly affected.

(6) Lack of granularity on the mobility management service. Mobility support for mobile user devices is provided as a per device management and not as per application basis.

(7) More complex network deployment. The use of centralized mobility anchors leads to a complex network deployment for all controls of traffic and mobility management is relegated to a single entity.

(8) Security. Centralized mobility anchors are susceptible for attacks.

The distributed mobility management (DMM) as a new architectural paradigm is a flat system architecture wherein the mobility anchors are distributed and is placed at the edge the access networks. The mobility management support for mobile user devices are distributed among these mobility anchors which are responsible for the 
distribution and control of data infrastructures. Mobile user devices can transfer its point of attachment between the mobility anchors [8].

\section{DMM based Multimedia Service Delivery}

The IETF working group is working on the standardization of DMM in order to address the limitations that are identified with the centralized mobility management architectures and they have come up with several solutions. That is, bringing the mobility anchors closer to the mobile user devices. One of the DMM based scheme is through the deployment of multiple HAs at the access network edge to serve as the mobile anchors. The mobile user device in this scheme uses multiple IP addresses which bind with the locally anchored address. The additional multiple IP addresses of the mobile user device serves as the CoA in the bindings [9].

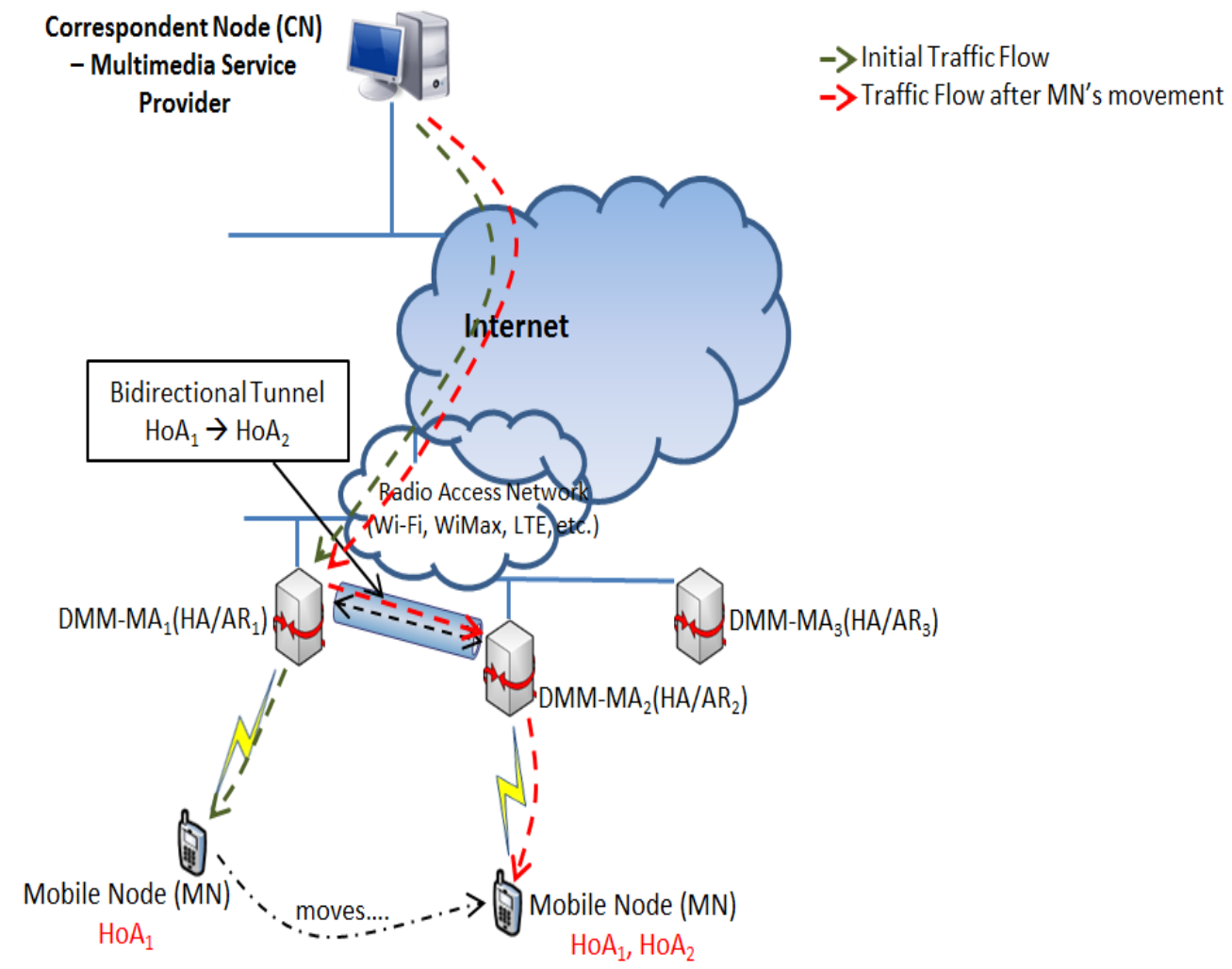

Figure 2. MIPv6 based DMM Multimedia Service Delivery

This scheme is depicted in Figure 2 indicating multiple HAs that are anchoring the traffic flow for the mobile user device. The bidirectional tunnels between HAs warrants the real-time multimedia service provision for the mobile user device whenever it moves from one network to the other. The multimedia service traffic flow from the $\mathrm{CN}$ initially traverses through the first DMM mobility anchor (DMM$\mathrm{MA}_{1}$ ) where the mobile user device is initially attached. Whenever the mobile user device transfers its point of attachment to DMM-MA $\mathrm{A}_{2}$, a new local address will be anchoring the mobile user device (i.e., CoA in the standard MIPv6) which will be used to make it reachable. The current multimedia service of $\mathrm{CN}$ provided to the mobile user device will then be tunneled from DMM-MA 1 to $\mathrm{DMM}^{\mathrm{D}} \mathrm{MA} \mathrm{A}_{2}$ (i.e., from $\mathrm{HoA}_{1}$ going to $\mathrm{HoA}_{2}$ ). This scheme has improved the handover latency since the mobility management has been localized and distributed among the mobility 
anchors which are very close to the mobile user devices. It is suitable for supporting the delivery of real-time multimedia applications to the users.

In Figure 3, the use of network-based DMM utilizing the PMIPv6 is depicted. The network-based distributed mobility solutions are divided into two subclasses: (1) the fully distributed scheme, wherein both the control and data planes are managed by the mobility anchors; and (2) the partially distributed scheme, the data and control planes are separated, and only the data plane is distributed by the mobility anchors. In a fully distributed scheme, each access router (AR) implements both the functions of LMA and MAG. The AR serves as an LMA that anchors and routes multimedia service traffic to the mobile user device and serves as a MAG that receives the tunneled multimedia service traffic to the user. Whereas, in a partially distributed scheme, the control plane is managed by a particular mobility management entity (MME) (e.g., as in 3GPP networks), while the data plane is distributed by the mobility anchors.

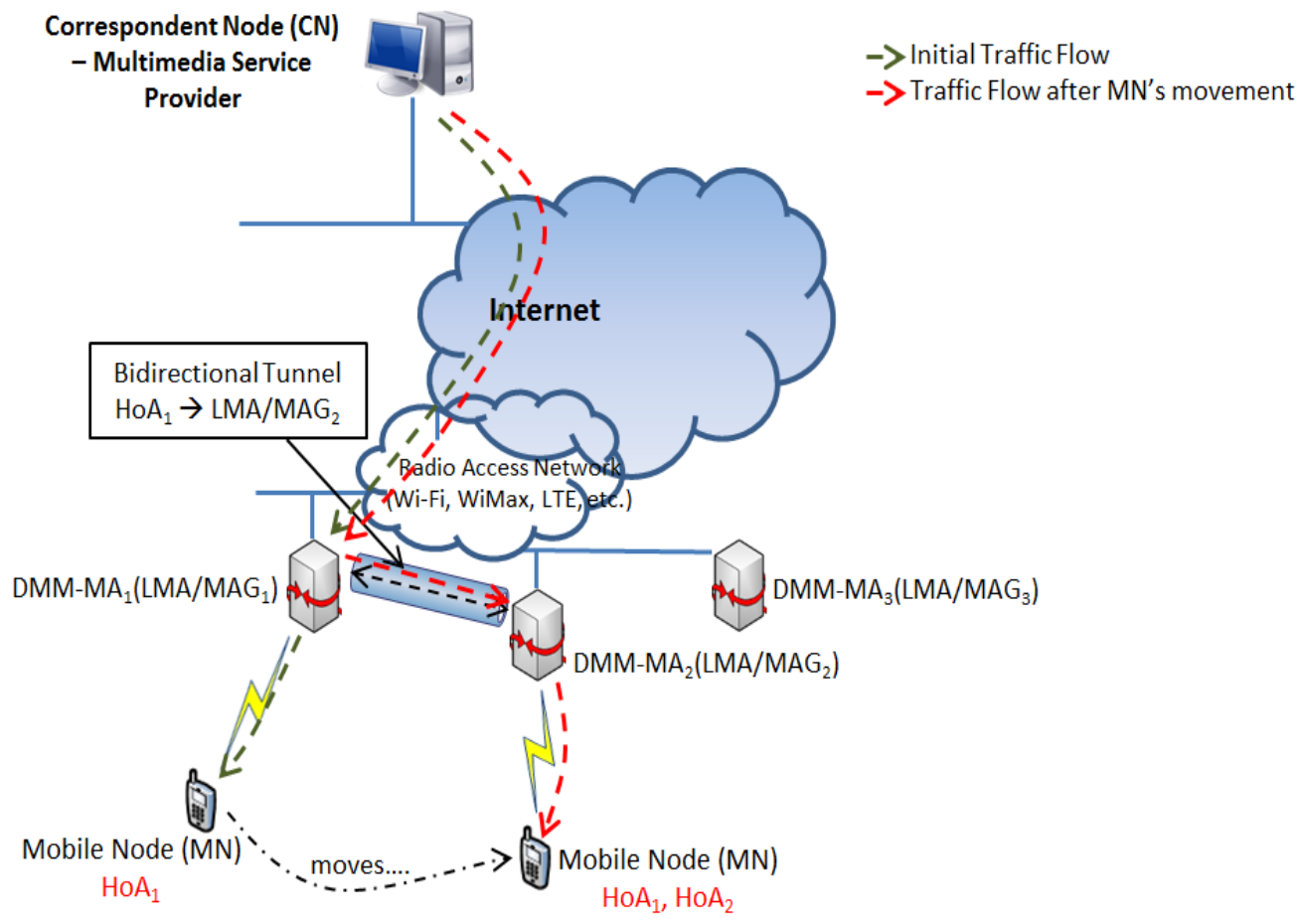

Figure 3. Generic Network-based DMM Multimedia Service Delivery

Multimedia service traffic is routed optimally through PMIPv6 based DMM paradigm since data traffic are distributed while the control on the connectivity of the mobile user devices are localized by a single centralized entity. This scheme is suitable for supporting real-time interactive video applications. 


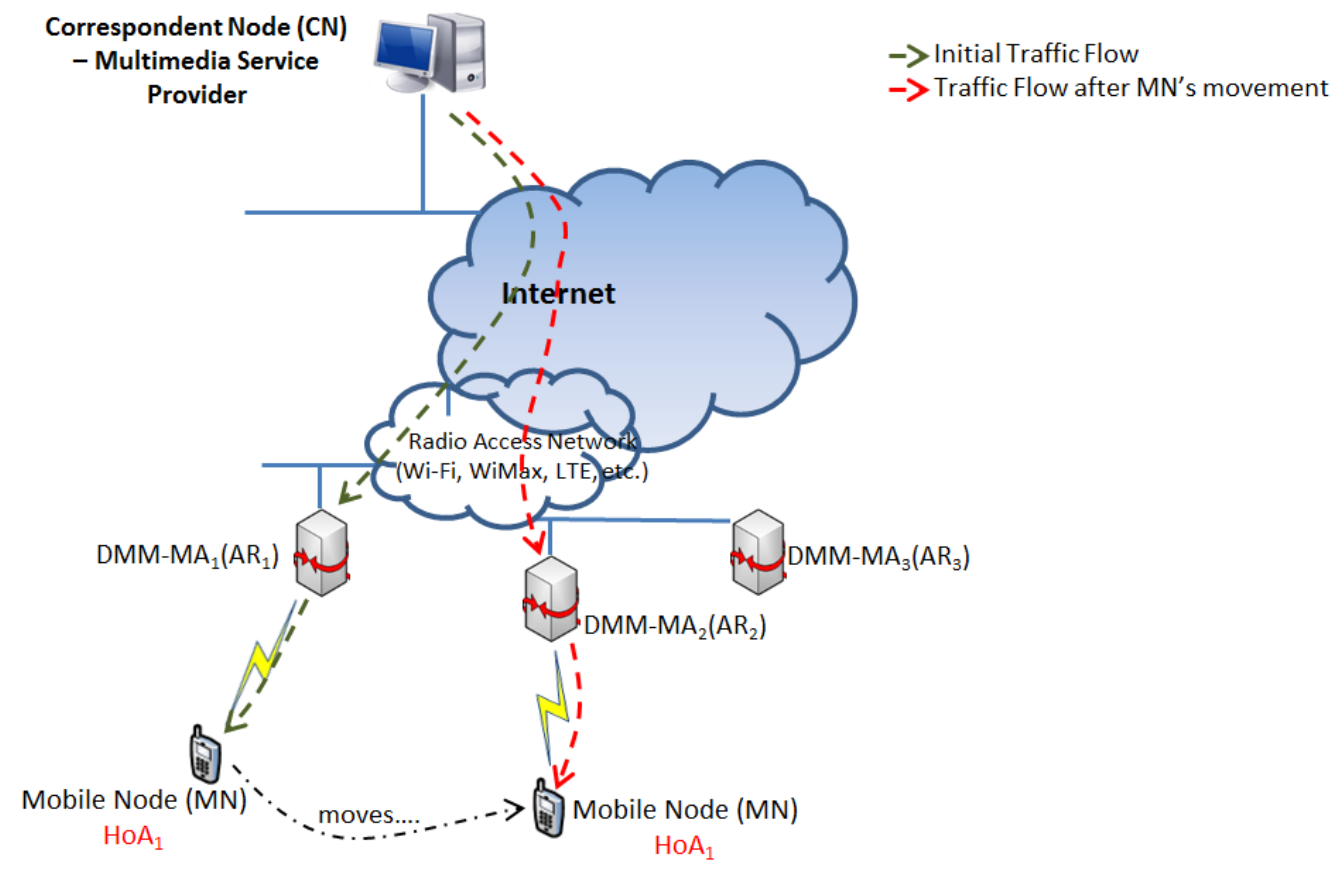

Figure 4. Routing based DMM Multimedia Service Delivery

The routing based DMM utilizes an intradomain protocol that internally advertises the obtained IP address of the mobile user device as it attaches to an AR as shown in Figure 4. This intradomain protocol refers to as the border gateway protocol (BGP). The new AR looks for the IP address with the host name of the mobile user device whenever it moves into its domain. A reverse lookup procedure is then performed by the new AR in order to confirm that the IP address is already associated with the mobile user device' host name and a routing update is then performed. Finally, A BGP update message is sent by the new AR containing the IP address of the mobile user device to other existing routers.

\section{Analysis of the PMIPv6 based DMM Multimedia Service Delivery}

The Internet of today is comprised of the integrated heterogeneous wireless networks with overlapping access points $[10,11,12]$. The cooperation among these wireless networks is essentially important for an efficient and seamless handover of connectivity for a mobile user device in order to obtain a quality of service (QoS) multimedia service provision. The use of hierarchical network systems that employs centralized mobility management is found to be limited in delivering a real-time multimedia service to the Internet users. In this regard, the DMM paradigm in delivering multimedia service to the users has been introduced.

In order to achieve such requirements, this paper analyzes the use of a PMIPv6 based DMM multimedia service delivery to the Internet users. The proposed scheme is outlined in Figure 5, wherein the data plane and control plane infrastructures for multimedia services have been separated. The control plane is managed by the mobile control anchor (DMM-MCA) which is responsible for the handling the network access control mechanisms such as mobility management, session management, etc.). The DMM-MCA manages the data traffic flow of multimedia services ensuring that QoS is received by the Internet users. 


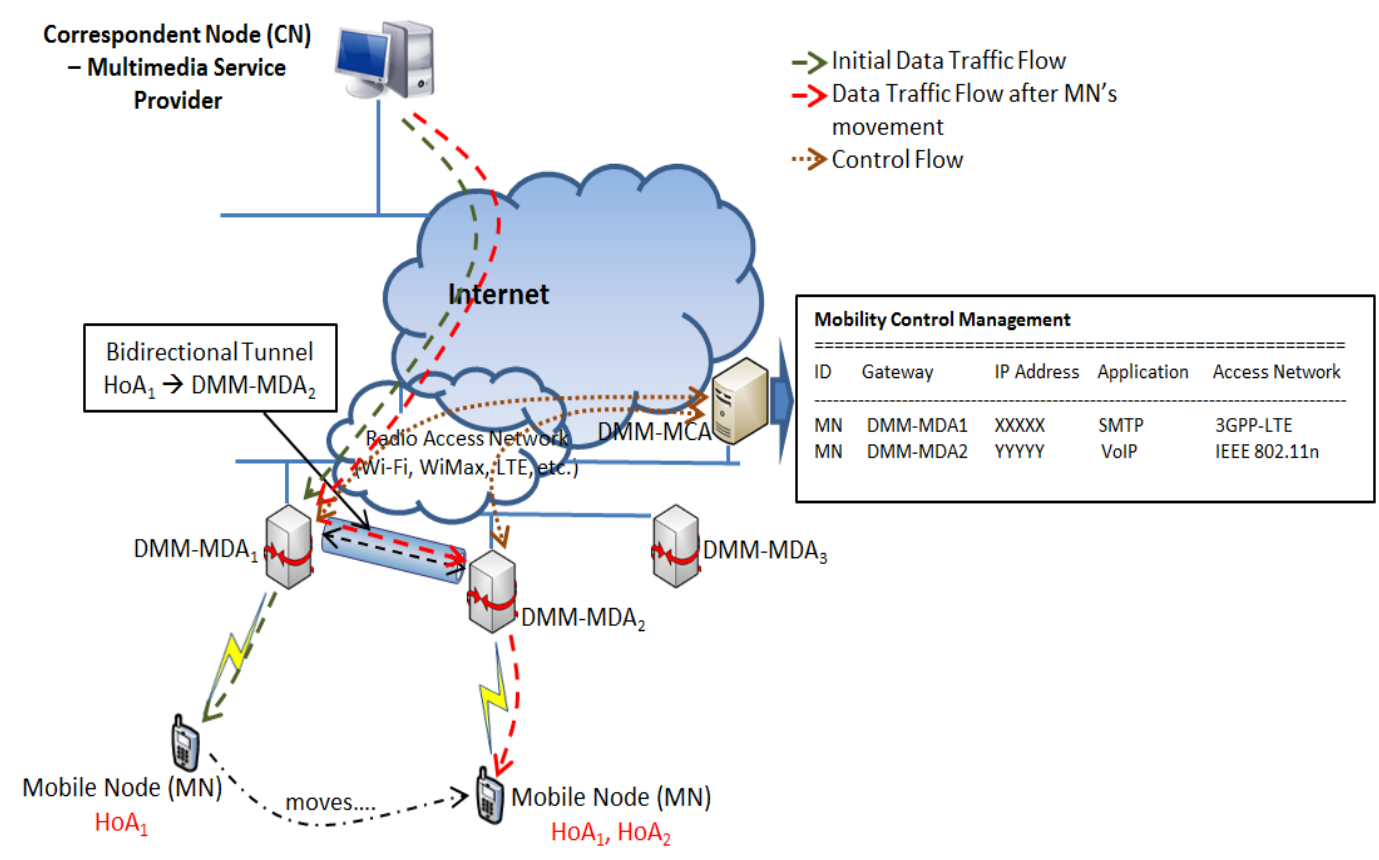

Figure 5. PMIPv6 based DMM Multimedia Service Delivery

The DMM mobile data anchor (DMM-MDA) is responsible for the delivery of multimedia service traffic to the Internet users. The mobile user device may attach to one of the available mobile data anchors and the DMM-MCA manages its address allocation and mobility support. The traffic flow will be determined by the DMM-MCA and multimedia service traffic will be tunneled by the previous DMM-MDA to the current DMM-MDA wherein the mobile user device is attached. Data traffic can traverse on an optimal route since it no longer goes back to the network core due to the separation from its control plane, thus, makes the delivery of multimedia service distributed.

\section{Conclusion}

This paper has presented an analysis of a PMIPv6 based DMM multimedia service delivery across the integrated heterogeneous wireless networks. Considering the large volume of multimedia traffic, the scheme addresses the identified issues of scalability, suboptimal routing, reliability, higher packet loss rate, signaling overhead, lack of granularity on the mobility management service, more complex network deployment, and security. The separation of the data plane and control plane infrastructures in providing mobility support for mobile user devices has provided a seamless real-time multimedia service to the Internet users. The delivery of multimedia service to the users was optimized due to the employment of mobility data anchors that are at the edge of the wireless access networks.

\section{Acknowledgments}

This research was supported by Basic Science Research Program through the National Research Foundation of Korea (NRF) funded by the Ministry of Science, ICT and future planning (2015R1A2A2A03002851). 


\section{References}

[1] CISCO, "Cisco Visual Networking Index: Forecast and Methodology, 2016-2021", White Paper, https://www.cisco.com/c/en/us/solutions/collateral/service-provider/visual-networking-indexvni/complete -white-paper-c11-481360.html, (2017) September.

[2] P. Bertin, S. Bonjour and J. Bonnin, "Distributed of centralized mobility", In Proceedings of IEEE Global Telecommunications Conference (GLOBECOM 2009), IEEE, (2009), pp. 1-6.

[3] H. A. Chan, H. Yokota, J. Xie, P. Seite and D. Liu, "Distributed and Dynamic Mobility Management in Mobile Internet: Current Approaches and Issues", Journal of Communications, Academy Publisher, vol. 6, no. 1, (2011), pp. 4-15.

[4] S. Gundavelli, K. Leung, V. Devarapalli, K. Chowdhury and B. Patil, "Proxy mobile IPv6", Internet Engineering Task Force (IETF), RFC 5213, (2008) August.

[5] C. J. Bernardos, M. Gramaglia, L. M. Contreras, M. Calderon and I. Soto, "Network-based Localized IP mobility Management: Proxy Mobile IPv6 and Current Trends in Standardization", Journal of Wireless Mobile Networks, Ubiquitous Computing, and Dependable Applications (JoWUA)(Special issue: Advances in Wireless Mobile and Sensor Technologies), vol. 1, no. 2/3, (2010), pp. 16-35.

[6] C. Perkins, D. Johnson and J. Arkko, "Mobility Support in IPv6", Internet Engineering Task Force (IETF), RFC 6275, ISSN: 2070-1721, (2011) July.

[7] H. Chan, "Requirements of Distributed Mobility Management", IETF Internet-Draft, (2012) July.

[8] J. C. Zúñiga, "Distributed Mobility Management: A Standards Landscape", IEEE Communications Magazine, vol. 51, no. 3, (2013) March, pp. 80-7.

[9] B. Sarikaya, "Distributed Mobile IPv6", IETF Internet-Draft, (2012) February.

[10] Y. Zhang, H. -H. Chen and M. Guizani, "Cooperative Wireless Communications", Wireless Networks and Mobile Communications Series, Boca Raton, FL: Auerbach Publications, Taylor \& Francis Group, (2009).

[11] W. Zhuang and M. Ismail, "Cooperation in Wireless Communication Networks", IEEE Wireless Communications, vol. 19, no. 2, (2012) April, pp. 10-20.

[12] L. Cai, "User Cooperation in Wireless Networks", IEEE Wireless Communications, vol. 19, no. 2 (2012) April, pp. 8-9. 
International Journal of Grid and Distributed Computing Vol. 10, No. 10 (2017) 